\title{
Intracellular trafficking of retroviral vectors: obstacles and advances
}

\author{
JL Anderson and TJ Hope \\ Department of Cell and Molecular Biology, Feinberg School of Medicine, Northwestern University, Chicago, IL, USA
}

\begin{abstract}
Retroviruses are efficient vehicles for delivering transgenes in vivo. Their ability to integrate into the host genome, providing a permanent imprint of their genes in the host, is a key asset for gene therapy. Furthermore, the lentivirus subset of retroviruses can infect nondividing as well as dividing cells. This expands the cell types capable of gene therapy, driving the development of lentiviral vectors. However, the precise mechanisms used by different retroviruses to efficiently deliver their genes into cell nuclei remains largely unclear. Understanding these molecular mechanisms may reveal features to improve the efficacy of current retroviral vectors. Moreover, this
\end{abstract}

Keywords: lentivirus; HIV; MLV; import; PIC; RTC; review

\section{Introduction}

When any microorganism infects cells, they must negotiate a complex cellular environment to survive and efficiently replicate progeny for continuing infection. Therefore, when looking for biological vehicles to deliver and express genes in hosts for gene therapy, microbes that efficiently enter and express their genes in a sustained manner while minimizing immune recognition serve as useful tools. Retroviruses are quite adept at surviving in such hostile environments to replicate. They can infect dividing ${ }^{1,2}$ and, in the case of lentiviruses, nondividing cells, ${ }^{3-5}$ efficiently trafficking through the cytoplasm to the nucleus for integration and gene expression to make progeny virions. Furthermore, their ability to integrate their DNA into the host genome provides a permanent imprint of the viral genes that are not lost through cell division. Retroviruses also commonly use methods to limit immune recognition, enabling long-lasting infections to be established in the host. For gene therapy vectors, these are important considerations for survival of the transgene in vivo. With such aptitude for delivering and expressing viral genes, retroviruses have thus received much attention for development into gene therapy vehicles.

Since simple retroviruses were initially developed into gene therapy vectors, this field has rapidly expanded into creating lentiviral vectors due to their wider target cell range. Retroviral vectors have overall yielded great

Correspondence: Dr TJ Hope, Department of Cell and Molecular Biology, Feinberg School of Medicine, Northwestern University, Chicago, IL 606113093, USA knowledge may expose elements pliable to other gene therapy vehicles to improve their in vivo performance and circumvent the biosafety concerns of using retroviral vectors. Therefore, the mechanisms underlying the early trafficking of retroviral vectors in host cells are reviewed here, as understood from studying the native retroviruses. Events after virus entry up to nuclear delivery of the viral cDNA are discussed. Cellular obstacles faced by these retroviral vectors and how they advance beyond these barriers is emphasized.

Gene Therapy (2005) 12, 1667-1678. doi:10.1038/

sj.gt.3302591

success for efficiently delivering genes or elements in vitro and in vivo. ${ }^{6}$ However, these vectors have a crucial safety issue (reviewed in Cockrell and Kafri, ${ }^{6}$ Pages and Bru, ${ }^{7}$ Anson $^{8}$ and Yi et al ${ }^{9}$ ). Their capacity to induce oncogenesis through retroviral insertion and generate new replication competent retroviruses has met with damaging results in vivo and complicates their current use in humans. ${ }^{10,11}$ Refining current retroviral vectors to improve biosafety and infection efficiency across more cell types will expand their use in vitro and in vivo. Furthermore, understanding how retroviruses efficiently infect host cells may reveal new features pliable to gene therapy vectors for better transgene delivery in vivo. This review therefore canvasses the early intracellular trafficking of retroviral vectors following viral entry to nuclear delivery. For those less acquainted with the topic, a general overview of retroviruses, retroviral vectors and the cell cytoskeleton precedes the discussion of vector trafficking, which is divided into cytoplasmic trafficking to the nucleus and nuclear import. Host cell factors that restrict retroviruses during these early trafficking events are further considered. As lentiviral vectors offer greater use due to their larger cell range, and are well characterized in the case of HIV-1, they form much of the discussion here.

\section{Retroviruses}

Retroviruses are classified by their ability to copy their RNA genome to a DNA intermediate using the viral enzyme, reverse transcriptase (RT). This process, termed reverse transcription, takes place during viral replication in host cells. Retroviral virions contain two copies of the 
positive sense, viral RNA genome within the viral protein core, surrounded by a cell membrane-derived envelope. Retroviral replication in target cells begins with binding of the virion envelope glycoprotein to the reciprocal cell surface receptor/s (Figure 1). With most retroviruses, the virion membrane fuses with the cell plasma membrane, releasing the internal core of the virion into the cell cytoplasm. The viral RNA genome in the core is reverse transcribed into cDNA within the confines of a viral reverse transcription complex (RTC). The completed viral cDNA intermediate in a matured complex termed the preintegration complex (PIC) sub-

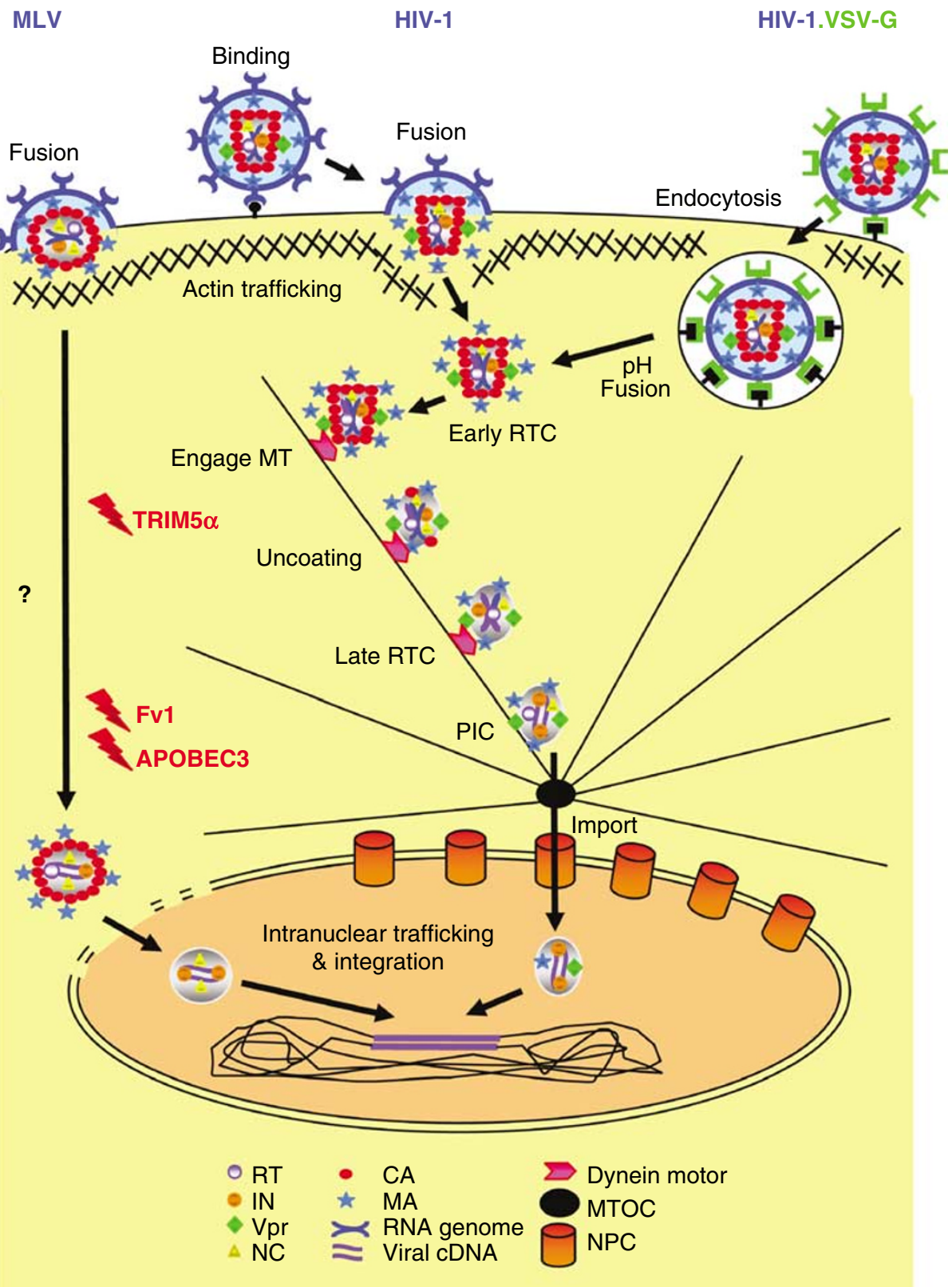

Figure 1 The early intracellular trafficking of retroviruses. This model proposes early replication events for MLV, HIV-1 and HIV-1 pseudotyped with a $p H$-dependent envelope-like VSV-G. The internal core of incoming virions are deposited in the cell cytoplasm after entry by fusion between the viral envelope and plasma membrane (left and middle panels) or endocytosis if pseudotyped with a pH-dependent envelope protein-like VSV-G (right panel). Cores entering at the plasma membrane encounter actin-dependent events, like penetrating the cortical actin barrier (middle panel) that is bypassed by endocytic entry (right panel). For HIV-1 and similar retroviruses, reverse transcribing, capsid cores bind dynein motors and traffic along microtubules towards the nucleus. During trafficking, the capsid is shed as reverse transcription proceeds. Cellular factors, like proteins aiding import and integration, are recruited to the RTC as it evolves into a PIC. At the nuclear envelope, viral cDNA in the PIC traffics through NPCs into the nucleus using multiple viral and cellular factors and may further traffic inside the nucleus for integration into favorable chromosome regions. For MLV (left panel), where information is scarce, the viral cores engage the actin cytoskeleton early but the RTCS retain the CA shell enroute to the nucleus. At the nuclear membrane, $C A$ and MA disassemble as the MLV complexes enter the nucleus during mitosis, where temporary dissolution of the nuclear envelope (indicated by broken lines) apparently permits access to cellular DNA for integration. During cytoplasmic trafficking, retroviruses are susceptible to (a) hypermutation and degradation if APOBEC3 is present in incoming virions, and (b) target cell TRIM5 $\alpha$ or Fv1, which bind and block susceptible cores before reverse transcription or integration respectively. The early trafficking of retroviral vectors is presumably similar to their original retroviruses, but removing viral proteins and/or nucleic acid elements may readily change the trafficking model presented here. 
sequently translocates into the nucleus and the viral cDNA integrates into the cellular genome. This results in a permanent copy of the viral genetic material incorporated in the host cell (Figure 1). The integrated viral DNA, termed a provirus, is subsequently transcribed and the mRNA translated to express the viral proteins. These viral proteins assemble at the cell plasma membrane with two copies of the full-length viral mRNA and bud off as immature virions. Subsequent processing of viral proteins within virions creates the mature, infectious particles capable of infecting new target cells (reviewed in Freed ${ }^{12}$ ).

All retroviruses express the structural group-specific antigen (Gag), polymerase (Pol) and envelope (Env) genes, but are further described as simple or complex due to additional genes. Simple retroviruses, typified by murine leukemia virus (MLV), just contain the three characteristic structural genes. In contrast, complex retroviruses like the lentiviruses, human immunodeficiency virus (HIV), and simian immunodeficiency virus (SIV), contain additional regulatory and accessory genes. The ability to pseudotype retroviruses with different envelope proteins, substitute pathogenic or nonessential genes for transgenes, and package the viral RNA using viral structural proteins expressed from separate plasmids allows these viruses to be used as vehicles for delivering transgenes.

\section{Retroviral vectors}

Since initial work with simple retroviruses like MLV to develop retroviral vectors, numerous vector systems based on simple and complex retroviruses now exist with various features, advantages and disadvantages too abundant to review here (discussed in Cockrell and Kafri, ${ }^{6}$ Pages and $\mathrm{Bru}^{7}$ and Delenda ${ }^{13}$ ). However, their general attributes remain the same. Retroviral vector systems commonly include the packaging, envelope and transgene vector components. The packaging plasmid or cell line provides the viral Gag and Pol structural proteins necessary to produce infectious virions. The envelope plasmid expresses the envelope protein incorporated into virion surfaces for entry into target cells. Envelope proteins that infect ubiquitous cell types (eg VSV-G or Ebola glycoprotein) or target-specific tissues (eg HIV-1 Env) may be used here. Finally, the transgene vector produces the RNA encoding the transgene that is packaged into vector virions. This RNA contains all the cis-acting elements necessary for virion packaging of the transgene RNA and subsequent reverse transcription, integration and expression of the transgene in target cells.

Separating these viral components during virus production should theoretically restrict virus infection to a single round in target cells, as the vector mRNA does not encode all the proteins to make new virions for subsequent infection. Although in practice, recombination between vector component/s or with endogenous retroviruses can generate undesirable replication-competent retrovirus. ${ }^{6,7,13}$ To avoid such deleterious recombination, all unnecessary retroviral sequence is deleted from the packaging, envelope and vector constructs. By deleting nonessential sequence from the transgene vector, large transgenes can also be packaged. For instance, complex lentiviral vectors can deliver up to 7-9 $\mathrm{kb}$ of extra sequence, demonstrating their utility in delivering and expressing large genes or elements (reviewed in Cockrell and Kafri ${ }^{6}$ ).

\section{Cell cytoskeleton}

To understand the early trafficking of retroviral vectors in target cells, first considering the intracellular environment these vectors must negotiate for successful trafficking is beneficial. The cell cytoplasm is a viscous compartment containing a scaffold of filaments termed the cytoskeleton. The cytoskeleton serves numerous roles, ranging from structural support to cell migration to trafficking cargo within the dense cell cytoplasm for such important processes as mitosis. The filaments of the cytoskeleton divide into three types: actin filaments, microtubules and intermediate filaments that are near 7,25 and $10-12 \mathrm{~nm}$ in diameter, respectively. These filaments are dynamic and can be readily assembled and disassembled according to molecular cues. Given the reported influence of actin filaments and microtubules on incoming retroviral trafficking, both filament types are discussed here. However, more detailed reviews of all three cytoskeleton filaments are found elsewhere. ${ }^{14-17}$

\section{Actin microfilaments}

The smallest filaments of the cytoskeleton, actin filaments, are also termed microfilaments owing to their petite size (reviewed in Revenu et al ${ }^{16}$ and Alberts et $a l^{17}$ ). Actin filaments are comprised of actin monomers that, after binding ATP, have polymerized. Monomeric and polymerized forms of actin are termed globular (G)-actin and filamentous $(F)$-actin, respectively. Actin filaments have polarity, with actin monomers binding the plus, barbed end of the growing filament and dissociating from the minus, pointed end. When actin monomers associate and dissociate from the opposite ends of the actin filament at a similar rate, the filament is said to be treadmilling. Individual actin filaments can further assemble into parallel bundles or loose networks via crosslinking proteins. Branched actin filaments can also form by nucleation promoting factors like WASP activating the actin-related protein (Arp) 2/3 complex, which binds to the side of an actin filament and nucleates a new filament. ${ }^{17}$ The crosslinking and branching of actin filaments allow cells to form more elaborate structures, like microvilli, stress fibers, lamellipodia, ruffles, cortical actin and filipodia. ${ }^{16}$ Besides regulating cell shape, mobility and adherence, these structures may also influence the trafficking of invading retroviral vectors. For instance, the dense layer of branched actin filaments underlying the plasma membrane of most cells termed cortical actin can serve as a barrier to invading pathogens. ${ }^{18,19}$

Actin filaments function in determining cell shape, strength, migration, adhesion, contraction and trafficking. ${ }^{16,17}$ For trafficking, cargo can travel along actin filaments using the myosin family of motor proteins, which generally move toward the plus end of actin filaments while hydrolyzing ATP. Myosin V and myosin I bind and move cargo like endoplasmic reticulum and golgi vesicles, respectively, along actin filaments. ${ }^{20}$ Some pathogens like Listeria and vaccinia virus also stimulate 
actin filament polymerization for movement in the cell. ${ }^{19}$ Therefore, binding myosin to move along the actin network or stimulating actin polymerization both allow transport within cells, and are thus important to consider for retrovirus trafficking, as well as actin filament structures that may hinder trafficking.

\section{Microtubules}

The microtubule network of filaments is critical for movement both within the cell and by cell appendages (reviewed in Alberts et $a l^{17}$ ). For instance, microtubules underlie chromosome movement within cells during division and allow cilia and flagella to move. Microtubules are composed of $\alpha$ and $\beta$ tubulin dimers that assemble into hollow, cylindrical filaments while hydrolyzing GTP. Tubulin dimers can bind either end of a microtubule in vitro, but polymerise more rapidly to the plus end where the $\beta$ tubulin GTP is exposed. The wall of one microtubule can also form part of the wall of a second microtubule filament, giving rise to doublet or triplet microtubules. ${ }^{17}$ In animal cells, microtubules emanate from the centrosome, which is also called the microtubule organizing center (MTOC, reviewed in Alberts et $a l^{17}$ and Sodeik et $a l^{21}$ ). The plus ends of microtubules grow out from the MTOC during the cell cycle, nucleating from $\gamma$-tubulin, while the minus ends remain anchored at the MTOC. The MTOC lies in the cytoplasm adjacent to the nucleus during interphase and houses the centrioles. The centrioles duplicate before cell division and move to opposite sides of the cell during division for coordinating chromosome segregation. ${ }^{21}$ Microtubules are key for moving chromosomes, organelles and other cargo within the cell, using the dynein and kinesin families of motor proteins for movement (for reviews see Alberts et $a l^{17}$ and Mallik and Gross ${ }^{22}$ ). Dyneins move cargo toward the minus end of microtubules, towards the MTOC that is retrograde transport. In contrast, kinesins move cargo toward the plus end of microtubules, away from the MTOC that is anterograde transport. Both dyneins and kinesins hydrolyze ATP during movement. These motor proteins allow for the rapid transport of cargo within cells, such as moving vesicles along the long axons of neurons to the tip via kinesins. ${ }^{17,22}$ Importantly, viruses such as herpes simplex virus type $\mathrm{I}^{23}$ adenovirus ${ }^{24,25}$ and $\mathrm{HIV}-1^{26}$ also latch onto dynein motors and use the microtubule network to traffic from the cell periphery toward the nucleus in cultured cells. Hence, microtubules and their molecular motors likely play an important role for efficient retroviral trafficking through the dense cell cytoplasm in vivo.

\section{Cytoplasmic trafficking}

\section{Early actin trafficking}

The ability to pseudotype retroviral vectors with envelope proteins from different sources has greatly expanded the repertoire of cells that retroviral vectors can enter to deliver transgenes. ${ }^{27}$ However, the choice of envelope protein may further influence the trafficking of viral cores upon entry into the cytoplasm. This is inferred by studies of early events in HIV-1 replication. ${ }^{28}$

The HIV-1 envelope protein (Env), like most retroviral envelope proteins, promotes fusion between virion and cell plasma membranes, depositing the virion core at the periphery of the cytoplasm (Figure 1). ${ }^{29}$ The HIV-1 accessory protein, Nef, further improves HIV-1 infection early in replication ${ }^{30}$ but only when virions enter cells using envelope proteins that fuse at the plasma membrane, like HIV-1 Env. If virions are pseudotyped with $\mathrm{pH}$-dependent envelope proteins deriving from VSV-G or Ebola, which enter cells via endocytosis and fuse in the cytoplasm after endosome acidification releasing the virion core (Figure 1), Nef no longer stimulates HIV-1 infection. $^{31}$ Recent work suggests this phenomenon maybe explained by how each entry route penetrates the cortical actin barrier underlying the plasma membrane. ${ }^{28}$ This study found disrupting cortical actin in epithelial cells by various actin depolymerising drugs compensated for Nef in improving HIV-1 infection when entering at the plasma membrane, but not by endocytic entry. $^{28}$ This suggests Nef performs a function in HIV-1 replication after plasma membrane entry that is mimicked by depolymerising actin, but how? Nef is incorporated into virions, remains with the viral core after entry into target cells, complexes with actin in cells and interacts with proteins involved in actin cytoskeleton rearrangement like Vav and PAK (discussed in Campbell et $\left.a l^{28}\right)$. This places Nef at the viral core in a position to perhaps remodel the actin cytoskeleton to facilitate HIV-1 infection. Thus, maybe viral cores deposited at the plasma membrane are less able to penetrate the cortical actin barrier without accessory proteins like Nef to rearrange actin for penetration. In contrast, perhaps virion cores entering the cytoplasm via endosomes bypass cortical actin more efficiently using this endogenous cell mechanism. As Nef improves HIV-1 infection between virion fusion and reverse transcription, ${ }^{28,32}$ this also suggests viral cores traffic past cortical actin before reverse transcription completes. Therefore, the envelope protein influences whether virion cores are deposited at the plasma membrane or deeper inside the cell cytoplasm via endosomes and thus, subsequent barriers like cortical actin that must be traversed for trafficking to the nucleus (Figure 1). As envelope proteins entering via endosomes seem more able to penetrate cortical actin, these proteins may therefore be better choices for delivering retroviral vectors to target cells rather than envelopes fusing at the plasma membrane.

While the actin cytoskeleton may act as a barrier to incoming retroviral vectors at the plasma membrane, ${ }^{28}$ separate studies infer the actin network also plays a positive role in early retroviral trafficking. ${ }^{33-35}$ For instance, the actin cytoskeleton can aid the clustering of HIV-1 CD4 receptor and CXCR4 coreceptor, facilitating HIV-1 infection. ${ }^{35}$ Fractionation studies also suggest HIV-1 RTCs rapidly associate with the actin cytoskeleton via the viral matrix (MA) protein, promoting functional RTCs to evolve. ${ }^{33}$ Blocking the cellular Arp2/3 complex that nucleates branched actin filaments can also impair HIV-1 and SIV infection. ${ }^{34}$ Hence, HIV-1 and SIV may use Arp2/3 to manipulate the actin cytoskeleton and improve infection, perhaps by propelling viral cores in the cytoplasm analogous to other pathogens using Arp2/ 3 for movement. ${ }^{19}$ MLV also uses the actin cytoskeleton early in viral infection ${ }^{36}$ but is not affected by Arp2/3 inhibition, ${ }^{34}$ suggesting MLV modulates actin by a different mechanism to Arp2/3 nucleation. Finally, pseudotyping HIV-1 with VSV-G to enter cells by endosomes eliminated the impact of actin microfila- 
ments ${ }^{33}$ and Arp2 $/ 3^{34}$ on HIV-1 infection. Hence, these studies indicate the actin cytoskeleton can enhance retroviral infection when entering at the plasma membrane by a mechanism bypassed with endocytic entry.

Microscope studies showing microtubule-independent motion of HIV-1 cores at the cell periphery after entry with the HIV-1 Env protein ${ }^{26}$ also supports actinmediated trafficking for incoming retroviral cores. The sensitivity of this HIV-1 trafficking to an actin depolymerising drug suggests the actin network underlies this short-range motion. This motion possibly reflects retroviral cores trying to penetrate cortical actin at the cell periphery or trafficking to another region of the peripheral cytoplasm for ensuing infection, such as linking to microtubules for efficient transport to the nucleus. $^{26}$ However, direct evidence showing HIV-1 cores trafficking on or through actin filaments in situ and what mechanism underlies this transport awaits demonstration. Why some studies find the actin cytoskeleton to facilitate viral infection ${ }^{33-35}$ while others consider actin to be a barrier ${ }^{28}$ remains unclear. Perhaps technical differences between studies or diverse functions of the actin cytoskeleton during early trafficking explain the discrepancy. Reconciling the positive and negative roles of the actin cytoskeleton in early retrovirus trafficking may allow current retroviral vectors to be refined to enhance their trafficking in different cell types. Ultimately, these studies hint at an actin-based trafficking event for virion cores entering at the plasma membrane that is avoided by endocytic entry, suggesting endocytic entry is a less complicated method to deliver retroviral vectors into cells.

\section{Uncoating, RTCs and PICs}

Following fusion of the virion membrane with either the cell plasma membrane or internal endosome membrane, the viral core is released into the cell cytoplasm (Figure 1). Viral cores are formed within virions during maturation, after budding from the producer cell (reviewed in Freed $^{37}$ ). During maturation, the viral Gag precursor protein is cleaved by the viral protease into its subunit proteins: MA, capsid (CA), nucleocapsid (NC) and in the case of lentiviruses, p6. MA forms a layer underlying the virion membrane while CA assembles into the casing of the core. The shape of this CA casing differs between retroviruses, aiding their classification. The core houses the viral genome complexed with $\mathrm{NC}$, viral RT and integrase (IN) enzymes plus cellular factors like tRNA essential for replication in target cells. When deposited into target cells, the RNA genomes in released cores are reverse transcribed into double-stranded cDNA, in a complex now termed a reverse transcription complex (RTC). When reverse transcription completes and the viral cDNA is ready for import into the nucleus, the complex is now termed a preintegration complex (PIC, see Figure 1). ${ }^{37}$ However, little is known about the process where the core evolves into an RTC and then a PIC, what factors are involved and where discrete steps occur in the cell cytoplasm.

For MLV, electron microscope (EM) analysis indicates MLV RTCs contain CA, MA and NC in the cytoplasm leading up the nuclear pores. ${ }^{38}$ Hence, these MLV Gag proteins seem to persist with RTCs in the cytoplasm. IN and RT are also detected in purified, cytoplasmic MLV complexes. ${ }^{39}$ In the vicinity of the nuclear envelope, these complexes disrupt and only NC and IN are detected in the nucleus, ${ }^{38}$ suggesting $\mathrm{MA}$ and $\mathrm{CA}$ are shed for nuclear entry. Similarly, the RTC/PIC of the complex animal retrovirus, human foamy virus (HFV), also reaches the nuclear vicinity as a structured CA, indicative of Gag proteins persisting with the core in the cytoplasm. ${ }^{40}$

In contrast, the maturation of HIV-1 cores appears more complex, beginning with a poorly characterized process of uncoating in the cytoplasm where viral CA protein is shed from the core. Where uncoating occurs in the cytoplasm is controversial. While some believe the viral CA immediately disassembles from the core after entry into the cytoplasm based on purified RTC/PIC preparations, ${ }^{41-43}$ others have evidence that the CA shell persists longer in the cytoplasm ${ }^{26,44}$ (see below). Furthermore, the role of reverse transcription in uncoating remains unclear. For instance, does CA loss from HIV-1 cores allow reverse transcription to proceed or could progressive reverse transcription, which expands viral nucleic acid trapped inside the core, force the core to burst, CA to be shed and the RTC to mature? Electron microscopy shows HIV-1 particles stimulated by free nucleotides to undergo endogenous reverse transcription within the virion also lose the CA shell of the core, ${ }^{45}$ supporting the theory reverse transcription forces CA shells to burst. Whatever the explanation, uncoating and reverse transcription seem intimately linked as HIV-1 CA mutations can impair reverse transcription ${ }^{46}$ and in situ or purified HIV-1 RTCs are elongated, unstructured and dissimilar to conical cores, ${ }^{26,47}$ consistent with uncoating.

Aside from shedding CA protein, the presence of MA and NC in maturing HIV-1 RTCs/PICs is also controversial, with opposing results between studies. ${ }^{26,41-43}$ However, reports that MA phosphorylation regulates late reverse transcript production ${ }^{48,49}$ and NC chaperones reverse transcription (reviewed elsewhere ${ }^{50}$ ) suggests MA and NC stay with HIV RTCs for atleast part of their cytoplasmic journey to impart their effects on reverse transcription. Along with the viral RT and IN enzymes, specialized viral proteins like the Vpr and Nef accessory proteins also remain with HIV-1 cores deposited in the cytoplasm. ${ }^{26,42,51}$ While Nef seems important for cores entering at the plasma membrane to interact with the actin cytoskeleton as discussed above, Vpr acts later in replication by influencing trafficking into the nucleus (considered below). Finally, HIV-1 RTCs/PICs also seem to recruit cellular proteins during their evolution in the cell. For instance, purified HIV-1 RTCs/PICs also contain cellular Ku70 and Ku90 nonhomologous end-joining proteins plus the HMG I(Y), LEDGF and BAF cellular proteins ${ }^{52-54}$ whose recruitment to HIV-1 PICs likely aids integration upon nuclear entry.

Overall, these studies collectively suggest that incoming retroviral cores undergo a series of compositional changes after entry, as reverse transcription progresses and the complex traffics to the nucleus for integration. Defining what these discrete changes are, where they occur in the cytoplasm, their timing and what factors are involved may reveal critical information to improve these cytoplasmic events for current retroviral vectors, increasing their efficacy in vivo.

However, major issues continue to obstruct studies of retroviral core maturation. Firstly, the poor stability of retroviral cores, typified by the sensitivity of 
HIV-1 conical CAs to detergents, may result in components being stripped from cores during sample extraction or other treatments, obscuring the study of authentic cores. Secondly, external components of the core may mask underlying components when studied by microscopy, adding to discrepancy. Thirdly, as recent studies indicate most HIV-1 cores rapidly traffic to the nuclear envelope after infection, ${ }^{26}$ time points at which cores are analyzed may complicate results. Hence, studying cores at different times postinfection should discriminate between cores at different maturation stages. Finally, reports that many HIV-1 virions enter cells nonproductively, ${ }^{55}$ raises the biggest issue of whether results pertain to authentic or nonproductive cores. Even though short viral harvests can curb this issue by maximizing the infectivity of virus used in experiments, the presence of nonproductive cores still complicates the study of authentic cores. Therefore, without appropriate methods to discriminate between authentic and nonproductive cores, it is difficult to address whether biochemical or microscopic results pertain to authentic, productively infecting cores or largely represent aberrant cores.

A recent study seems to be overcoming many of these pitfalls by examining HIV-1 cores in situ using fluorescence microscopy. ${ }^{26}$ Labeling HIV-1 cores with GFP-Vpr, which is packaged into virions and stays with the core in the cell cytoplasm, allows viral cores to be tracked in situ. Staining virion membranes with a fluorescent DiD dye can discriminate between HIV-1 productively entering the cell by plasma membrane fusion, where the DiD virus membrane is lost, versus cores entering through nonproductive endocytosis, where the virion membrane remains trapped with the core in the endosome. Finally, authentic reverse transcribing cores are identified by their incorporation of a fluorescent nucleotide during reverse transcription. Using these methods, authentic HIV-1 cores reverse transcribing in the cell were tracked and two types of RTC were identified: HIV CA (p24 ${ }^{\mathrm{CA}}$ )positive and -negative complexes. ${ }^{26}$ Both RTC types contained the HIV-1 MA protein (p17 ${ }^{\mathrm{MA}}$ ) consistent with some biochemical studies. ${ }^{41,43}$ While the p24 ${ }^{\mathrm{CA}}$-positive RTCs may represent defective complexes incapable of further infection, it is equally possible these p24 ${ }^{\mathrm{CA}}$ positive and -negative RTCs represent an early and later step in RTC maturation enroute to the nucleus. Recent work investigating HIV-1 restriction in owl monkey cells supports the notion early p24 ${ }^{\mathrm{CA}}$ HIV-1 RTCs exist in the cytoplasm. ${ }^{44}$ As wildtype or hyperstable viral CAs can saturate HIV-1 restriction in target cells but unstable CAs or improperly processed Gag cannot, ${ }^{44}$ this indicates intact CA structures are recognized for restriction. Therefore, HIV-1 CA cores must remain intact for some period in the cell cytoplasm for restriction and thus, the p24 ${ }^{\text {CA }}$-positive RTCs identified in HIV-1 infected cells ${ }^{26}$ may be legitimate, early RTCs. Finally, both p24 ${ }^{\mathrm{CA}}$ positive and -negative RTCs associated with microtubules by microscopy, suggesting these RTCs associate with microtubules before the p24 ${ }^{\mathrm{CA}}$ casing is lost. ${ }^{26}$ Hence, this in situ work implies productive HIV-1 cores deposited in the cytoplasm begin reverse transcribing with a p24 ${ }^{\mathrm{CA}}$ case, associate with microtubules and uncoat this $\mathrm{p} 24^{\mathrm{CA}}$ casing as the RTC traffics to the nucleus (modeled in Figure 1). Hence, these innovative microscopic methods offer a novel perspective to study HIV-1 cores as they mature in the cell and may reveal many details so far eluded by conventional biochemical methods.

\section{Trafficking to the nucleus}

The cell cytoplasm is viscous and filled with cytoskeleton networks, organelles and cellular vesicles, which likely hinder particles moving through the cytoplasm by simple diffusion. ${ }^{56}$ Instead, cytoskeleton filaments can be used for rapid and directed cytoplasmic trafficking. When retroviral cores are deposited in the cytoplasm typically at the plasma membrane following fusion, they must then move to the nucleus for integration into the host cell DNA. Nonretroviral pathogens like herpes simplex virus type 1 (HSV-1 $)^{23}$ and adenovirus ${ }^{24,25}$ traffic to the nucleus by latching onto dynein motor proteins and moving along the microtubules. Recent data indicates HIV-1 also uses dynein motors and the microtubule network for trafficking to the nucleus as disrupting either element-impaired HIV-1 cores from moving to the nucleus. ${ }^{26}$ Furthermore, HIV-1 cores were imaged trafficking along microtubules to the nucleus in live cells. ${ }^{26}$ Interestingly, HIV-1 IN, which comprises RTCs/PICs, also interacts with yeast microtubule-associated proteins, consistent with HIV-1 interacting with the microtubule network to traffic in the cell cytoplasm. ${ }^{57}$

Besides HIV-1, the human foamy retrovirus (HFV) also uses the microtubule network and dynein-dynactin motors to traffic from the cell periphery to the nucleus in infected cells. ${ }^{40,58}$ These HFV cores accumulate at the pericentrosomal region of the MTOC as structured CAs, mediated by the HFV Gag protein. ${ }^{40}$ The MTOC is the origin of emanating microtubules and likely to be a weigh station for all viruses on their way to the nucleus, as demonstrated for adenovirus. ${ }^{59}$ Although, how viruses move from the MTOC to the nucleus remains to be determined. Ultimately, defining MTOC localization as a prerequisite for nuclear entry and the responsible viral motifs will provide new insight into retroviral trafficking into the nucleus. Novel features to enhance the trafficking of current gene therapy vectors may further be highlighted.

\section{Trafficking into the nucleus}

Successful trafficking of viral cores to the nuclear envelope does not guarantee productive infection, as the PIC must still permeate this nuclear envelope barrier for integration and gene expression in the nucleus. The double membrane, nuclear envelope of eukaryotic cells separates the nuclear and cytoplasmic contents in interphase cells. Molecules move between the interphase nucleus and cytoplasm via large, macromolecular, nuclear pore complexes (NPCs) spanning the nuclear envelope (reviewed in Weis ${ }^{60}$ and Fahrenkrog and Aebi $\left.{ }^{61}\right)$. Small molecules up to $40-60 \mathrm{kDa}$ can diffuse through NPCs to gain access to the alternate compartment. Conversely, larger cargoes use nuclear localization signals (NLSs) and nuclear export signals, aided by the cellular RanGTP gradient, to actively transport through NPCs into the nucleus and cytoplasm, respectively. ${ }^{60}$ For active import into the nucleus, NLS associated with the cargo recruit transport proteins like members of the importin $\beta$ superfamily for trafficking (reviewed in Weis $\left.{ }^{60}\right)$. The ability of incoming retroviral PICs to cross 
the nuclear membrane is determined by the origin of the retrovirus (Figure 1) and differs between lentiviral and simple retroviral vectors.

For the simple MLV retroviruses, early experiments revealed that the cell cycle controls the nuclear import of MLV PICs, which only enter the nucleus during mitosis. $^{1,2}$ Therefore, these simple retroviruses appear unable to traverse through NPCs during interphase and thus cannot infect nondividing cells. Instead, nuclear entry is restricted to mitosis where temporary dissolution of the nuclear membrane for impending cell division is thought to give MLV access to the nuclear DNA (Figure 1). Why MLV cannot traffic into nondividing cell nuclei via NPCs is unclear. Recent data with chimeric MLV and HIV-1 Gag proteins indicates that the MLV CA prevents PICs from trafficking into the nucleus of nondividing cells. ${ }^{62}$ Therefore, perhaps MLV PICs lack NLS or fail to expose NLS due to the MLV CA, preventing their NPC import. Alternatively, the MLV CA may sterically obstruct NPC import to preclude infection of nondividing cells.

In contrast, lentiviruses such as HIV-1 infect dividing and nondividing cells, like terminally differentiated macrophages. ${ }^{3-5}$ This implies lentiviruses penetrate the nuclear envelope through NPCs to access nuclear DNA in nondividing cells. Support for HIV-1 PICs importing through NPCs is seen in early work showing HIV-1 PICs use an active, energy-dependent mechanism for nuclear import. ${ }^{4}$ Furthermore, recent work suggests HIV-1 cDNA import and integration is independent of mitosis in dividing cells, and may in fact prefer the interphase step of the cell cycle, consistent with PIC import via NPCs. ${ }^{63}$ Expressing VSV MA protein in cells to diminish NPC transport also impairs the nuclear import of HIV-1 cDNAs, further implicating NPCs in HIV-1 PIC import ${ }^{64}$ (Figure 1). Traditionally, the nuclear import of HIV-1 PICs has been attributed to seemingly redundant, NLSs present in the viral Vpr, MA and IN proteins that comprise PICs (for reviews see Nisole and Saib ${ }^{29}$ and Dvorin and Malim $^{52}$ ). However, more recent studies suggest other factors may underlie PIC import, including the viral central DNA 'flap' structure formed during reverse transcription, ${ }^{65}$ the viral CA protein ${ }^{62}$ and the cellular proteins, importin $7^{66}$ and nucleoporin $98 .^{64}$ The potential role of these elements in HIV-1 import are discussed in detail elsewhere $29,52,62,64,66$ but the controversy surrounding their function suggests multiple factors aid HIV import through nuclear pores.

It may seem perplexing, HIV-1 CA could facilitate PIC nuclear import given previous biochemical and microscopic studies clearly indicate CA is uncoated from the HIV-1 RTC in the cytoplasm prior to nuclear import. ${ }^{26,41-43}$ Therefore, how might CA promote nuclear import if it has already been shed from the PIC? It is possible HIV-1 CA may direct viral RTCs on a cytoplasmic pathway before being shed that leads to efficient NPC trafficking. Alternatively, the shedding of HIV-1 CA from the core may enable steric changes for HIV-1 cDNA to move through NPCs or expose underlying NLSs in the viral RTC that allow nuclear import via NPCs. Hence, loss of HIV-1 CA during RTC maturation could indirectly promote import through NPCs. However, it should be noted that the role of CA in HIV-1 PIC import remains controversial as $\mathrm{CA}$ was only recently implicated in PIC import ${ }^{62}$ and is yet to be shown to act as or contain a transferable NLS. Importantly, more research in this area could explain the long-standing enigma of how lentiviruses like HIV-1 import their cDNA into nondividing cells and is thus worthy of our attention. The role of HIV-1 MA in PIC import may also seem at odds with a biochemical study implying MA is lost from viral RTCs containing cDNA in the cytoplasm. ${ }^{42}$ However, complete MA loss from RTCs is controversial as separate biochemical and in situ microscopic studies clearly track HIV-1 MA in viral RTCs, ${ }^{26,41,43}$ up to the nuclear envelope microscopically. ${ }^{26}$ Furthermore, HIV-1 MA is important for PIC import in nondividing cells like macrophages and mutating both NLSs in MA blocks HIV-1 cDNA import in macrophages. ${ }^{67}$ So most data indicate some HIV-1 MA remains with cytoplasmic HIV-1 RTCs/PICs ${ }^{26,41,43}$ and the MA NLSs aid nuclear import in nondividing cells. ${ }^{67,68}$ Although, MA clearly is not the primary determinant for importing HIV-1 PICs as the MA NLSs are dispensable for import in dividing cells. ${ }^{67}$

Finally, how HIV-1 PICs move through NPCs despite their large size likely exceeding the limit for active NPC transport $^{52}$ also remains unknown. Perhaps, like the large Balbiani ring ribonucleoprotein, ${ }^{69}$ the lentiviral PIC also unfolds at the nuclear envelope allowing the viral cDNA to move through NPCs, similar to herpesvirus and adenovirus. $^{70}$ Therefore, despite the numerous studies exploring HIV-1 PIC import, the viral and cellular factors involved and their mechanism/s of action remain unclear. No sole determinant of import has been reproducibly identified among studies and instead, this quagmire hints multiple factors govern HIV1 cDNA import. Defining the role of these factors among cell types, particularly in nondividing cells, should reveal new tools to improve the nuclear import of current gene therapy vectors in vivo.

Aside from the lentiviruses, other retroviruses like avian sarcoma virus (ASV) and foamy virus (FV) also enter the nucleus of nondividing cells, albeit at lower efficiency to dividing cells (for reviews see Nisole and $\mathrm{Saib}^{29}$ and $\mathrm{Katz}^{71}$ ). Like HIV-1, an NLS in ASV IN protein is speculated to underlie PIC import. ${ }^{72}$ In contrast, the factors that translocate FV PICs into the nucleus are unknown. However, cytoplasmic FV cores traffic to the MTOC, ${ }^{40}$ which lies close to the nuclear envelope, perhaps aiding nuclear entry. Therefore, understanding these import processes maybe as beneficial as studying lentiviruses for finding new tools to enhance gene therapy vector import in nondividing cells.

\section{Avoiding host cell restrictions}

When any microorganism invades a host, the ability to counter host cell defense mechanisms and manipulate cellular processes to favor the pathogen are critical elements for survival and persistence of the microorganism. Retroviruses are no exception to this battle between pathogens and their host. In fact, recent developments in understanding host cell factors that restrict retroviral infection has garnered enormous interest in these restriction factors (reviewed in Goff ${ }^{73}$ and Bieniasz ${ }^{74}$ ). Of late, two new types of cellular restriction factors have been identified that can interfere with the early, intracellular trafficking of retroviral vectors discussed 
here: the APOBEC3 and the Fv1/TRIM5 $\alpha$ restriction factors (Figure 1). These classes of restriction factors are considered below but more detailed reviews are found elsewhere. ${ }^{30,73-75}$ Interferons are also well established to perturb early retroviral infection, lowering proviral DNA, ${ }^{76-79}$ but are not discussed here due to space constraints.

The importance of the Apolipoprotein B mRNAediting catalytic polypeptide-like 3 (APOBEC3) factors for restricting retroviral replication first materialized in 2002 when APOBEC3G (initially termed CEM15) was identified as blocking HIV-1. ${ }^{80}$ Since this seminal study, multiple human APOBEC3 family members and their homologues in other species are reported to block diverse retroviruses (reviewed in Harris and Liddament ${ }^{75}$ ). The APOBEC3 proteins belong to an enzyme family that all deaminate cytidine (C) bases to uracil (U) in DNA or RNA. ${ }^{81,82}$ The human APOBEC3 members act during reverse transcription by deaminating $\mathrm{C}$ to $\mathrm{U}$ in single-stranded viral cDNA, which induces guanine to adenine mutations in the complementary cDNA strand. ${ }^{83}$ To function, human APOBEC3G is packaged into virions in producer cells but blocks viral replication in target cells (discussed in Harris and Liddament ${ }^{75}$ ), most likely by degrading the viral cDNA between late reverse transcription and integration. ${ }^{84}$ In defense, the lentiviruses use their viral infectivity factor (Vif) accessory protein to prevent APOBEC3 packaging into virions in producer cells and circumvent restriction in target cells (reviewed in Anderson and $\mathrm{Hope}^{30}$ and Harris and Liddament $^{75}$ ). Importantly, as APOBEC3 must be present in the virus-producing cell for packaging into virions and then restriction in target cells, this negative role of APOBEC3 can be readily eliminated from retroviral gene therapy vectors by producing virions in cells lacking APOBEC3. Therefore, this early restriction to retroviral infection can be readily evaded by carefully choosing the virus-producing cells.

The second category of cellular factors that can restrict early retroviral replication in target cells are Fv1 and TRIM5 $\alpha$ (reviewed in $^{73,74}$ ). These cellular factors were first identified by Friend virus susceptibility gene 1 $(\mathrm{Fv} 1),{ }^{85}$ a restriction factor in mouse cells that blocks specific strains of MLV. ${ }^{86}$ Fv1 encodes a protein similar to mouse and human ERV-L endogenous retrovirus Gag ${ }^{87,88}$ but the mechanism of Fv1 restriction remains unclear. MLV sensitivity to Fv1 restriction is determined by amino acid 110 in the viral CA protein. ${ }^{89,90}$ High virus inoculums can saturate Fv1 restriction but expressing Gag protein in target cells before infection cannot, suggesting Fv1 targets incoming viral CA cores. ${ }^{73,74}$ Fv1 restriction blocks MLV between reverse transcription and integration in the nucleus. ${ }^{91}$ Hence, Fv1 may restrict MLV by binding the viral CA core in the cytoplasm, preventing subsequent integration in the nucleus. Therefore, when delivering genes to mouse cells, the species of retroviral vector must be carefully chosen to ensure the vector is not susceptible to Fv1 restriction, which would impair vector efficacy.

Following Fv1, restriction factor 1 (Ref1) and lentivirus susceptibility factor 1 (Lv1) were then identified in human and non-human primate cells respectively. ${ }^{92-94}$ Similar to Fv1, restriction by Ref1 and Lv1 could be saturated and the retroviral CA protein governed virus susceptibility to these factors. ${ }^{92-95}$ However, Ref1 and
Lv1 both block retroviral replication before reverse transcription, ${ }^{92,95}$ thus acting at an earlier replication step than Fv1, which restricts between reverse transcription and integration. ${ }^{91}$ Ref1 and Lv1 block a different range of retroviruses, which generally exclude retroviruses normally found in the same species as the restriction factor (reviewed in Goff $^{73}$ ). The recent discovery that TRIM $5 \alpha^{96}$ underlies Ref1 and Lv1 restriction in different species ${ }^{96-98}$ has made stunning progress in understanding these restriction factors.

TRIM5 $\alpha$ belongs to a large family of TRIM proteins named for their tripartite motifs: the RING, B-box and coiled-coil sequences. These family members also contain a variable $C$-terminus which includes a fourth motif called the B30.2 or SPRY domain in TRIM5 $\alpha .{ }^{99}$ Studies with human and rhesus TRIM5 $\alpha$ chimeras indicate this SPRY domain determines which retrovirus species are restricted by TRIM5 $\alpha .^{100-102}$ Separate work in owl monkey cells supports this conclusion. These cells contain an unusual TRIM5-Cyclophilin A (TRIMCypA)-fusion protein, arising from retrotransposition of a CypA pseudogene into the TRIM5 $\alpha$ locus, replacing the TRIM5 $\alpha$ SPRY domain for CypA. ${ }^{103,104}$ TRIM-CypA restricts HIV-1 infection in owl monkey cells. ${ }^{103,104}$ The CypA portion of TRIM-CypA also binds HIV-1 CA in vitro, ${ }^{104}$ like human CypA binds HIV-1 CA. ${ }^{105}$ This suggests the CypA portion of TRIM-CypA binds HIV-1 CA in vivo in place of the lost SPRY domain while the $\mathrm{N}$-terminal TRIM motifs restrict virus infection. Hence, these studies infer the C-terminal SPRY domain of TRIM5 $\alpha$ determines binding to different retroviral CA proteins while the N-terminal tripartite motifs cause restriction. How TRIM5 $\alpha$ blocks retroviral infection before reverse transcription so far remains unclear. A clue to TRIM5 $\alpha$ function may lie in other members of the TRIM family, who localize to specific cellular regions and can assemble into structures like the cytoplasmic speckles of TRIM5. ${ }^{99}$ Therefore, perhaps TRIM5 $\alpha$ similarly forms aggregates in the cytoplasm around incoming retroviral cores through binding the viral CA shell. This could potentially trap the viral cores in the cytoplasm, preventing their ability to uncoat or traffic to other regions of the cytoplasm necessary for reverse transcription. Alternatively, recruiting TRIM $5 \alpha$ to the viral CA core may target the incoming cores for degradation before reverse transcription.

Clearly, much remains to be learned about the molecular mechanisms underlying TRIM5 $\alpha$ and Fv1 restriction and why the CAs of some retroviral species escape restriction while others are sensitive. Delineating these molecular mechanisms will advance our knowledge of this species-specific battle between host and pathogen, and guide our choice of retroviral systems to deliver transgenes into different hosts. For instance, as human TRIM5 $\alpha$ restricts N-tropic MLV and equine infectious anemia virus (EIAV) ${ }^{73}$ using retroviral Gag proteins deriving from these viruses to deliver a vector into human cells maybe problematic given their sensitivity to human TRIM $5 \alpha$ restriction. However, TRIM5 $\alpha$ restriction can be saturated using an excess of incoming viral cores. ${ }^{98}$ Therefore, if viral vectors like EIAV currently under development ${ }^{106,107}$ are to be used for human gene therapy, higher titers of vector will likely be necessary to saturate the TRIM $5 \alpha$ restriction for efficient infection of human target cells. Finally, understanding 
how TRIM5 $\alpha$ and Fv1 block early retroviral replication $^{91,92,95}$ may also yield new knowledge about these early replication steps while exposing new antiviral targets to prevent early retroviral infection in vivo. Clearly, an exciting future awaits this research field.

\section{Future directions}

When retroviruses and retroviral vectors enter target cells, they must overcome numerous obstacles to deliver their genes into the nucleus for expression. Cellular barriers like cortical actin, cytoplasm viscosity, the nuclear envelope and cellular restriction factors all play a part in obstructing the passage of and thus defending the cell against invading retroviruses. To advance through these barriers, retroviruses have evolved numerous tactics. For instance, binding microtubule motors for rapid directed trafficking to the nucleus, ${ }^{26,40,58}$ using multiple signals for moving through NPCs into the nucleus ${ }^{29,52}$ and encoding accessory proteins like HIV-1 Vif to counter cellular restriction factors ${ }^{80}$ all enable retroviruses to efficiently traffic to the nucleus for gene delivery. Therefore, for optimal performance, retroviral vectors should contain all the elements to efficiently progress past these early cellular obstacles for maximal gene delivery in the nucleus. To achieve this, all the retroviral motifs allowing efficient gene delivery in different cell types must first be identified.

While some early steps of retrovirus replication leading to efficient gene delivery are well understood, such as the enzymatic steps of reverse transcription and integration, other events like the maturation and trafficking of viral cores are poorly characterized. Furthermore, the retroviral motifs underlying many trafficking steps are either unknown or controversial, like the motifs for HIV-1 nuclear import. Recent studies are starting to progress understanding of these early trafficking events and thus, an updated model outlining how retroviruses pass cellular obstacles and deliver their genes to cell nuclei is presented (Figure 1).

Clearly much remains to be learned about the early journey of retroviral cores that culminates in gene delivery. Defining the trafficking pathways and viral motifs underlying virus movement to and through the nuclear envelope should most benefit the design of future gene therapy vectors, ensuring they contain all the elements for efficient trafficking in vivo. Focusing studies on the retroviruses that penetrate nondividing cell nuclei also seems wise. This may expose viral motifs transferable to other types of gene therapy vectors to expand their target cell range and perhaps avoid the use of retroviral vectors in vivo with their current biosafety setbacks. Recent work with mutated IN proteins $^{108}$ and characterizing integration sites ${ }^{109,110}$ hints retroviral cDNA also undergoes intranuclear trafficking, perhaps for efficient integration into specific regions of the cell genome-like actively transcribing genes. Defining such intranuclear trafficking may also reveal novel viral motifs to facilitate retroviral gene delivery. Delineating the exact mechanisms behind TRIM5 $\alpha$, Fv1 and APOBEC3 restriction is also a future priority as such knowledge will guide the choice of retroviral vectors to deliver transgenes in different host species. While biochemistry and genetics continue to drive understanding of early retrovirus replication, we feel microscopy also has an important future role. The behavior of authentic, reverse transcribing cores can be explored in live cells by microscopy, providing real-time details that may be lost in other analyses.

Therefore, retroviral vectors are efficient tools for delivering transgenes into an array of cell types. Understanding the molecular details behind their efficient trafficking should advance the design of future gene therapy vectors, generating a new wave of vectors with much wider therapeutic value.

\section{Acknowledgements}

This work was supported through the National Institutes of Health RO1 Grant AI47770 to TJ Hope.

\section{References}

1 Roe T, Reynolds TC, Yu G, Brown PO. Integration of murine leukemia virus DNA depends on mitosis. $E M B O J$ 1993; 12: 2099-2108.

2 Lewis PF, Emerman M. Passage through mitosis is required for oncoretroviruses but not for the human immunodeficiency virus. J Virol 1994; 68: 510-516.

3 Weinberg JB, Matthews TJ, Cullen BR, Malim MH. Productive human immunodeficiency virus type 1 (HIV-1) infection of nonproliferating human monocytes. J Exp Med 1991; 174: 1477-1482.

4 Bukrinsky MI et al. Active nuclear import of human immunodeficiency virus type 1 preintegration complexes. Proc Natl Acad Sci USA 1992; 89: 6580-6584.

5 Lewis P, Hensel M, Emerman M. Human immunodeficiency virus infection of cells arrested in the cell cycle. EMBO J 1992; 11: 3053-3058.

6 Cockrell AS, Kafri T. HIV-1 vectors: fulfillment of expectations, further advancements, and still a way to go. Curr HIV Res 2003; 1: 419-439.

7 Pages JC, Bru T. Toolbox for retrovectorologists. J Gene Med 2004; 6 (Suppl 1): S67-S82.

8 Anson DS. The use of retroviral vectors for gene therapy - what are the risks. A review of retroviral pathogenesis and its relevance to retroviral vector-mediated gene delivery. Genet Vaccines Ther 2004; 2: 9.

9 Yi Y, Hahm SH, Lee KH. Retroviral gene therapy: safety issues and possible solutions. Curr Gene Ther 2005; 5: 25-35.

10 Hacein-Bey-Abina $\mathrm{S}$ et al. LMO2-associated clonal $\mathrm{T}$ cell proliferation in two patients after gene therapy for SCID-X1. Science 2003; 302: 415-419.

11 Check E. Gene therapy put on hold as third child develops cancer. Nature 2005; 433: 561.

12 Freed EO. HIV-1 and the host cell: an intimate association. Trends Microbiol 2004; 12: 170-177.

13 Delenda C. Lentiviral vectors: optimization of packaging, transduction and gene expression. J Gene Med 2004; 6 (Suppl 1): S125-S138.

14 Chang L, Goldman RD. Intermediate filaments mediate cytoskeletal crosstalk. Nat Rev Mol Cell Biol 2004; 5: 601-613.

15 Coulombe PA, Wong P. Cytoplasmic intermediate filaments revealed as dynamic and multipurpose scaffolds. Nat Cell Biol 2004; 6: 699-706.

16 Revenu C, Athman R, Robine S, Louvard D. The co-workers of actin filaments: from cell structures to signals. Nat Rev Mol Cell Biol 2004; 5: 635-646. 
17 Alberts B et al. Chapter 16: The Cytoskeleton. In: Gibbs S (ed). Molecular Biology of the Cell, 4th edn. Garland Science: New York, 2002, pp 907-982.

18 Marsh M, Bron R. SFV infection in CHO cells: cell-type specific restrictions to productive virus entry at the cell surface. J Cell Sci 1997; 110 (Part 1): 95-103.

19 Gruenheid S, Finlay BB. Microbial pathogenesis and cytoskeletal function. Nature 2003; 422: 775-781.

20 DePina AS, Langford GM. Vesicle transport: the role of actin filaments and myosin motors. Microsc Res Tech 1999; 47: 93-106.

21 Varmark H. Functional role of centrosomes in spindle assembly and organization. J Cell Biochem 2004; 91: 904-914.

22 Mallik R, Gross SP. Molecular motors: strategies to get along. Curr Biol 2004; 14: R971-R982.

23 Sodeik B, Ebersold MW, Helenius A. Microtubule-mediated transport of incoming herpes simplex virus 1 capsids to the nucleus. J Cell Biol 1997; 136: 1007-1021.

24 Kelkar SA, Pfister KK, Crystal RG, Leopold PL. Cytoplasmic dynein mediates adenovirus binding to microtubules. J Virol 2004; 78: 10122-10132.

25 Suomalainen $\mathrm{M}$ et al. Microtubule-dependent plus- and minus end-directed motilities are competing processes for nuclear targeting of adenovirus. J Cell Biol 1999; 144: 657-672.

26 McDonald D et al. Visualization of the intracellular behavior of HIV in living cells. J Cell Biol 2002; 159: 441-452.

27 Burns JC et al. Vesicular stomatitis virus G glycoprotein pseudotyped retroviral vectors: concentration to very high titer and efficient gene transfer into mammalian and nonmammalian cells. Proc Natl Acad Sci USA 1993; 90: 8033-8037.

28 Campbell EM, Nunez R, Hope TJ. Disruption of the actin cytoskeleton can complement the ability of Nef to enhance human immunodeficiency virus type 1 infectivity. J Virol 2004; 78: 5745-5755.

29 Nisole S, Saib A. Early steps of retrovirus replicative cycle. Retrovirology 2004; 1: 9.

30 Anderson JL, Hope TJ. HIV accessory proteins and surviving the host cell. Curr HIV/AIDS Rep 2004; 1: 47-53.

31 Chazal $\mathrm{N}$ et al. Human immunodeficiency virus type 1 particles pseudotyped with envelope proteins that fuse at low $\mathrm{pH}$ no longer require Nef for optimal infectivity. J Virol 2001; 75: 4014-4018.

32 Tobiume $\mathrm{M}$ et al. Nef does not affect the efficiency of human immunodeficiency virus type 1 fusion with target cells. J Virol 2003; 77: 10645-10650.

33 Bukrinskaya A, Brichacek B, Mann A, Stevenson M. Establishment of a functional human immunodeficiency virus type 1 (HIV-1) reverse transcription complex involves the cytoskeleton. J Exp Med 1998; 188: 2113-2125.

34 Komano J, Miyauchi K, Matsuda Z, Yamamoto N. Inhibiting the Arp2/3 complex limits infection of both intracellular mature vaccinia virus and primate lentiviruses. Mol Biol Cell 2004; 15: 5197-5207.

35 Iyengar S, Hildreth JE, Schwartz DH. Actin-dependent receptor colocalization required for human immunodeficiency virus entry into host cells. J Virol 1998; 72: 5251-5255.

36 Kizhatil K, Albritton LM. Requirements for different components of the host cell cytoskeleton distinguish ecotropic murine leukemia virus entry via endocytosis from entry via surface fusion. J Virol 1997; 71: 7145-7156.

37 Freed EO. HIV-1 replication. Somat Cell Mol Genet 2001; 26: 13-33.

38 Risco $\mathrm{C}$ et al. Intracellular transport of the murine leukemia virus during acute infection of NIH 3T3 cells: nuclear import of nucleocapsid protein and integrase. J Cell Sci 1995; 108 (Part 9): 3039-3050.

39 Fassati A, Goff SP. Characterization of intracellular reverse transcription complexes of Moloney murine leukemia virus. J Virol 1999; 73: 8919-8925.
40 Petit $\mathrm{C}$ et al. Targeting of incoming retroviral Gag to the centrosome involves a direct interaction with the dynein light chain 8. J Cell Sci 2003; 116: 3433-3442.

41 Miller MD, Farnet CM, Bushman FD. Human immunodeficiency virus type 1 preintegration complexes: studies of organization and composition. J Virol 1997; 71: 5382-5390.

42 Fassati A, Goff SP. Characterization of intracellular reverse transcription complexes of human immunodeficiency virus type 1. J Virol 2001; 75: 3626-3635.

43 Bukrinsky MI et al. Association of integrase, matrix, and reverse transcriptase antigens of human immunodeficiency virus type 1 with viral nucleic acids following acute infection. Proc Natl Acad Sci USA 1993; 90: 6125-6129.

44 Forshey BM, Shi J, Aiken C. Structural requirements for recognition of the human immunodeficiency virus type 1 core during host restriction in owl monkey cells. J Virol 2005; 79: 869-875.

45 Zhang H, Dornadula G, Orenstein J, Pomerantz RJ. Morphologic changes in human immunodeficiency virus type 1 virions secondary to intravirion reverse transcription: evidence indicating that reverse transcription may not take place within the intact viral core. J Hum Virol 2000; 3: 165-172.

46 Forshey BM, von Schwedler U, Sundquist WI, Aiken C. Formation of a human immunodeficiency virus type 1 core of optimal stability is crucial for viral replication. J Virol 2002; 76: 5667-5677.

47 Nermut MV, Fassati A. Structural analyses of purified human immunodeficiency virus type 1 intracellular reverse transcription complexes. J Virol 2003; 77: 8196-8206.

48 Kaushik R, Ratner L. Role of human immunodeficiency virus type 1 matrix phosphorylation in an early postentry step of virus replication. J Virol 2004; 78: 2319-2326.

49 Kiernan RE, Ono A, Englund G, Freed EO. Role of matrix in an early postentry step in the human immunodeficiency virus type 1 life cycle. J Virol 1998; 72: 4116-4126.

50 Bampi $\mathrm{C}$ et al. The chaperoning and assistance roles of the HIV1 nucleocapsid protein in proviral DNA synthesis and maintenance. Curr HIV Res 2004; 2: 79-92.

51 Forshey BM, Aiken C. Disassembly of human immunodeficiency virus type 1 cores in vitro reveals association of nef with the subviral ribonucleoprotein complex. J Virol 2003; 77: 4409-4414.

52 Dvorin JD, Malim MH. Intracellular trafficking of HIV-1 cores: journey to the center of the cell. Curr Top Microbiol Immunol 2003; 281: 179-208.

53 Llano $\mathrm{M}$ et al. LEDGF/p75 determines cellular trafficking of diverse lentiviral but not murine oncoretroviral integrase proteins and is a component of functional lentiviral preintegration complexes. J Virol 2004; 78: 9524-9537.

54 Lin CW, Engelman A. The barrier-to-autointegration factor is a component of functional human immunodeficiency virus type 1 preintegration complexes. J Virol 2003; 77: 5030-5036.

55 Andreadis $S$ et al. Toward a more accurate quantitation of the activity of recombinant retroviruses: alternatives to titer and multiplicity of infection. J Virol 2000; 74: 3431-3439.

56 Luby-Phelps K. Cytoarchitecture and physical properties of cytoplasm: volume, viscosity, diffusion, intracellular surface area. Int Rev Cytol 2000; 192: 189-221.

57 de Soultrait VR et al. HIV-1 integrase interacts with yeast microtubule-associated proteins. Biochim Biophys Acta 2002; 1575: $40-48$

58 Saib A et al. Nuclear targeting of incoming human foamy virus Gag proteins involves a centriolar step. J Virol 1997; 71: 1155-1161.

59 Bailey CJ, Crystal RG, Leopold PL. Association of adenovirus with the microtubule organizing center. J Virol 2003; 77: 13275-13287. 
60 Weis K. Regulating access to the genome: nucleocytoplasmic transport throughout the cell cycle. Cell 2003; 112: 441-451.

61 Fahrenkrog B, Aebi U. The nuclear pore complex: nucleocytoplasmic transport and beyond. Nat Rev Mol Cell Biol 2003; 4: 757-766.

62 Yamashita M, Emerman M. Capsid is a dominant determinant of retrovirus infectivity in nondividing cells. J Virol 2004; 78: 5670-5678.

63 Katz RA, Greger JG, Boimel P, Skalka AM. Human immunodeficiency virus type 1 DNA nuclear import and integration are mitosis independent in cycling cells. J Virol 2003; 77: 13412-13417.

64 Ebina $\mathrm{H}$ et al. Role of Nup98 in nuclear entry of human immunodeficiency virus type 1 cDNA. Microbes Infect 2004; 6: 715-724.

65 Zennou $\mathrm{V}$ et al. HIV-1 genome nuclear import is mediated by a central DNA flap. Cell 2000; 101: 173-185.

66 Fassati A et al. Nuclear import of HIV-1 intracellular reverse transcription complexes is mediated by importin 7. EMBO J 2003; 22: 3675-3685.

67 Haffar OK et al. Two nuclear localization signals in the HIV-1 matrix protein regulate nuclear import of the HIV-1 preintegration complex. J Mol Biol 2000; 299: 359-368.

68 Bukrinsky MI et al. A nuclear localization signal within HIV-1 matrix protein that governs infection of non-dividing cells. Nature 1993; 365: 666-669.

69 Daneholt B. Assembly and transport of a premessenger RNP particle. Proc Natl Acad Sci USA 2001; 98: 7012-7017.

70 Greber UF, Fassati A. Nuclear import of viral DNA genomes. Traffic 2003; 4: 136-143.

71 Katz RA, Greger JG, Skalka AM. Effects of cell cycle status on early events in retroviral replication. J Cell Biochem 2005; 94: 880-889.

72 Kukolj G, Katz RA, Skalka AM. Characterization of the nuclear localization signal in the avian sarcoma virus integrase. Gene 1998; 223: 157-163.

73 Goff SP. Retrovirus restriction factors. Mol Cell 2004; 16: 849-859.

74 Bieniasz PD. Intrinsic immunity: a front-line defense against viral attack. Nat Immunol 2004; 5: 1109-1115.

75 Harris RS, Liddament MT. Retroviral restriction by APOBEC proteins. Nat Rev Immunol 2004; 4: 868-877.

76 Avery RJ et al. Interferon inhibits transformation by murine sarcoma viruses before integration of provirus. Nature 1980; 288: 93-95.

77 Salzberg S, Shurtz R, Feder J, Aboud M. Effect of interferon on the penetration of murine leukemia virus and the binding of its genome RNA to polyribosomes at the early stage of infection. Arch Virol 1983; 78: 267-278.

78 Cremer I, Vieillard V, De Maeyer E. Retrovirally mediated IFNbeta transduction of macrophages induces resistance to HIV, correlated with up-regulation of RANTES production and down-regulation of C-C chemokine receptor-5 expression. J Immunol 2000; 164: 1582-1587.

79 Vieillard V, Lauret E, Rousseau V, De Maeyer E. Blocking of retroviral infection at a step prior to reverse transcription in cells transformed to constitutively express interferon beta. Proc Natl Acad Sci USA 1994; 91: 2689-2693.

80 Sheehy AM, Gaddis NC, Choi JD, Malim MH. Isolation of a human gene that inhibits HIV-1 infection and is suppressed by the viral Vif protein. Nature 2002; 418: 646-650.

81 Jarmuz A et al. An anthropoid-specific locus of orphan C to U RNA-editing enzymes on chromosome 22. Genomics 2002; 79: 285-296.

82 Harris RS, Petersen-Mahrt SK, Neuberger MS. RNA editing enzyme APOBEC1 and some of its homologs can act as DNA mutators. Mol Cell 2002; 10: 1247-1253.
83 Harris RS et al. DNA deamination mediates innate immunity to retroviral infection. Cell 2003; 113: 803-809.

84 Mariani $\mathrm{R}$ et al. Species-specific exclusion of APOBEC3G from HIV-1 virions by Vif. Cell 2003; 114: 21-31.

85 Lilly F. Susceptibility to two strains of Friend leukemia virus in mice. Science 1967; 155: 461-462.

86 Pincus T, Hartley JW, Rowe WP. A major genetic locus affecting resistance to infection with murine leukemia viruses. IV. Doseresponse relationships in $\mathrm{Fv}$-1-sensitive and resistant cell cultures. Virology 1975; 65: 333-342.

87 Best S, Le Tissier P, Towers G, Stoye JP. Positional cloning of the mouse retrovirus restriction gene Fv1. Nature 1996; 382 826-829.

88 Benit $\mathrm{L}$ et al. Cloning of a new murine endogenous retrovirus, MuERV-L, with strong similarity to the human HERV-L element and with a gag coding sequence closely related to the Fv1 restriction gene. J Virol 1997; 71: 5652-5657.

89 DesGroseillers L, Jolicoeur P. Physical mapping of the Fv-1 tropism host range determinant of $\mathrm{BALB} / \mathrm{c}$ murine leukemia viruses. J Virol 1983; 48: 685-696.

90 Kozak CA, Chakraborti A. Single amino acid changes in the murine leukemia virus capsid protein gene define the target of Fv1 resistance. Virology 1996; 225: 300-305.

91 Pryciak PM, Varmus HE. Fv-1 restriction and its effects on murine leukemia virus integration in vivo and in vitro. J Virol 1992; 66: 5959-5966.

92 Towers $\mathrm{G}$ et al. A conserved mechanism of retrovirus restriction in mammals. Proc Natl Acad Sci USA 2000; 97: 1229512299.

93 Cowan $\mathrm{S}$ et al. Cellular inhibitors with Fv1-like activity restrict human and simian immunodeficiency virus tropism. Proc Natl Acad Sci USA 2002; 99: 11914-11919.

94 Hofmann W et al. Species-specific, postentry barriers to primate immunodeficiency virus infection. I Virol 1999; 73 : 10020-10028

95 Owens CM, Yang PC, Gottlinger H, Sodroski J. Human and simian immunodeficiency virus capsid proteins are major viral determinants of early, postentry replication blocks in simian cells. I Virol 2003; 77: 726-731.

96 Stremlau M et al. The cytoplasmic body component TRIM5alpha restricts HIV-1 infection in Old World monkeys. Nature 2004; 427: 848-853.

97 Keckesova Z, Ylinen LM, Towers GJ. The human and African green monkey TRIM5alpha genes encode Ref1 and Lv1 retroviral restriction factor activities. Proc Natl Acad Sci USA 2004; 101: 10780-10785.

98 Hatziioannou Tet al. Retrovirus resistance factors Ref1 and Lv1 are species-specific variants of TRIM5alpha. Proc Natl Acad Sci USA 2004; 101: 10774-10779.

99 Reymond A et al. The tripartite motif family identifies cell compartments. EMBO J 2001; 20: 2140-2151.

100 Stremlau M, Perron M, Welikala S, Sodroski J. Species-specific variation in the B30.2(SPRY) domain of TRIM5\{alpha\} determines the potency of human immunodeficiency virus restriction. J Virol 2005; 79: 3139-3145.

101 Sawyer SL, Wu LI, Emerman M, Malik HS. Positive selection of primate TRIM5\{alpha\} identifies a critical species-specific retroviral restriction domain. Proc Natl Acad Sci USA 2005; 102: 2832-2837.

102 Yap MW, Nisole S, Stoye JP. A single amino acid change in the SPRY domain of human Trim5alpha leads to HIV-1 restriction. Curr Biol 2005; 15: 73-78.

103 Sayah DM, Sokolskaja E, Berthoux L, Luban J. Cyclophilin A retrotransposition into TRIM5 explains owl monkey resistance to HIV-1. Nature 2004; 430: 569-573.

104 Nisole S, Lynch C, Stoye JP, Yap MW. A Trim5-cyclophilin A fusion protein found in owl monkey kidney cells can restrict HIV-1. Proc Natl Acad Sci USA 2004; 101: 13324-13328. 
105 Luban J et al. Human immunodeficiency virus type 1 Gag protein binds to cyclophilins A and B. Cell 1993; 73: 1067-1078.

106 Waddington SN et al. Long-term transgene expression by administration of a lentivirus-based vector to the fetal circulation of immuno-competent mice. Gene Therapy 2003; 10: 1234-1240.

107 Gregory LG et al. Highly efficient EIAV-mediated in utero gene transfer and expression in the major muscle groups affected by Duchenne muscular dystrophy. Gene Therapy 2004; 11: 1117-1125.
$108 \mathrm{Lu} \mathrm{R}$ et al. Class II integrase mutants with changes in putative nuclear localization signals are primarily blocked at a postnuclear entry step of human immunodeficiency virus type 1 replication. J Virol 2004; 78: 12735-12746.

109 Schroder AR et al. HIV-1 integration in the human genome favors active genes and local hotspots. Cell 2002; 110: 521-529.

$110 \mathrm{Wu}$ X, Li Y, Crise B, Burgess SM. Transcription start regions in the human genome are favored targets for MLV integration. Science 2003; 300: 1749-1751. 